\title{
Social and Emotional Learning in Preschool Settings: A Systematic Map of Systematic Reviews
}

\author{
Dariush Djamnezhad ${ }^{1}$, Nadia Koltcheva ${ }^{2}$, Alma Dizdarevic ${ }^{3}$, Amila Mujezinovic $^{3}$, \\ Carla Peixoto ${ }^{4,5}$, Vera Coelho ${ }^{4}$, Mart Achten ${ }^{6}$, Erika Kolumbán ${ }^{7}$, Francisco Machado ${ }^{4}$ and \\ Björn Hofvander ${ }^{1,8,9 *}$
}

${ }^{1}$ LU-CRED, Child and Adolescent Psychiatry, Department of Clinical Sciences Lund, Lund University, Lund, Sweden, ${ }^{2}$ Department of Cognitive Science and Psychology, New Bulgarian University, Sofia, Bulgaria, ${ }^{3}$ Faculty of Special Education and Rehabilitation, University of Tuzla, Tuzla, Bosnia and Herzegovina, ${ }^{4}$ Department of Social and Behavioural Sciences, University Institute of Maia, ISMAl, Maia, Portugal, ${ }^{5}$ inED-Centre for Research and Innovation in Education, Porto, Portugal, ${ }^{6}$ Erasmus Brussels University of Applied Sciences and Arts, Brussels, Belgium, ${ }^{7} P e t o ̋$ András Faculty, Semmelweis University, Budapest, Hungary, ${ }^{8}$ Centre for Ethics, Law and Mental Health, Department of Psychiatry and Neurochemistry, Institute of Neuroscience and Physiology, The Sahlgrenska Academy at University of Gothenburg, Gothenburg, Sweden, ${ }^{9}$ Department of Forensic Psychiatry, Region Skåne, Sweden

\section{OPEN ACCESS}

Edited by:

Ricardo Primi,

Universidade São Francisco, Brazil

Reviewed by:

Xin Gong,

Central China Normal University,

China

Inmaculada Marín-López,

Universidad de Córdoba, Spain

*Correspondence:

Björn Hofvander

bjorn.hofvander@med.lu.se

Specialty section:

This article was submitted to Educational Psychology,

a section of the journal

Frontiers in Education

Received: 06 April 2021

Accepted: 07 July 2021

Published: 24 August 2021

Citation:

Djamnezhad D, Koltcheva $N$ Dizdarevic A, Mujezinovic A, Peixoto C, Coelho V, Achten M, Kolumbán E, Machado F and Hofvander B (2021)

Social and Emotional Learning in Preschool Settings: A Systematic Map of Systematic Reviews. Front. Educ. 6:691670. doi: $10.3389 /$ feduc.2021.691670
The preschool years presents an important opportunity to support children's social and emotional development. Social and emotional learning (SEL) programs in early childhood education and care (ECEC) have gained an increased interest due to its potential to improve child health and educational outcomes. We aimed to identify existing systematic reviews on universal, curriculum-based SEL interventions in ECEC settings (children aged 0 to 7 years), assess their risk of bias, synthesize the findings and identify knowledge gaps. We undertook a systematic literature search in seven different databases. Reviews of studies without control groups were excluded. Each abstract and full text article was assessed independently, and disagreements were solved in consensus. Relevant reviews were assessed for bias using the ROBIS tool. Of 4912 records identified through database searches, two systematic reviews met the inclusion criteria. Both reviews were assessed as having a high risk of bias. The results were used to summarize existing knowledge and knowledge gaps. In conclusion, SEL interventions in preschool settings must be considered knowledge gaps. There is a need for more high-quality primary studies and further systematic reviews that adhere to strict scientific methods and address the overwhelming heterogeneity in field, in terms of interventions, settings and outcomes.

Keywords: SEL, preschool, interventions, systematic review, universal

\section{INTRODUCTION}

Over the last decades, a vast amount of research has been accumulated worldwide regarding social and emotional skills development in children. The importance of these skills, sometimes referred to as non-cognitive skills, "soft" skills or character skills, to promote a healthy overall development is emphasized in numerous studies (e.g., Durlak et al., 2011; Jones et al., 2015; Bierman et al., 2016; Domitrovich et al., 2017; Eklund et al., 2018). Weissberg and colleagues (2015, p. 3) expressed a similar position when writing: "The past 20 years have witnessed an explosion of interest in social and emotional learning (SEL). Research reviews have documented the value of SEL programs and 
schools, families, and communities are partnering to promote the positive development and academic success of children and youth across the globe."

Early childhood is a pivotal period for the development of social and emotional skills (Jones and Bouffard, 2012; McClelland et al., 2017). Starting at birth, or even during the prenatal period, developmental foundations of social-emotional competence are perhaps foremost laid during the early childhood years (Prado and Dewey, 2014; Spencer et al., 2017). Early experiences strongly influence how young children begin to understand themselves and the world that surrounds them (Yates et al., 2008). To support the development of social and emotional skills during early childhood, internal and external factors have to be taken into account. Internal factors refer to children's characteristics such as temperament or personality. On the other hand, it is vital to provide children with an environment, i.e., family, school, social and cultural contexts, where they feel safe and secure in order to contribute to the social and emotional development (Yates et al., 2008; McClelland et al., 2017).

During the pre-school years, dramatic transformations occur in children's social skills, social reasoning, emotional understanding, emotional regulation, self-awareness, and self-control (Bierman and Motamedi, 2015). Over the past two decades, compelling evidence from longitudinal studies has shown the critical role that early childhood social and emotional skills play in children's school adjustment and academic achievement (e.g., Bierman et al., 2009; Denham et al., 2012; Nakamichi et al., 2021), as well as in other long-term life outcomes such as mental health, substance use, and criminal behavior (e.g., Moffit et al., 2011; Jones et al., 2015). As a response, a considerable growth in research, aiming to inform the development of interventions and policies that facilitate the growth of these skills and maximize children's well-being, has taken place (e.g., Bierman and Motamedi, 2015; Domitrovich et al., 2017; Eklund et al., 2018).

Within the field of socio-emotional skills, practitioners and researchers use different constructs to organize, define, and describe the research area (Berg et al., 2019). Among these constructs, SEL has served as an umbrella for a range of approaches and appears to have the largest and most rigorously evaluated evidence base (Goldberg et al., 2019). SEL is usually described as the process by which children and adults learn to understand and manage emotions, maintain positive relationships, make responsible decisions, set and achieve positive goals, feel and show empathy for others, as well as improve their capacity to solve problems effectively (Weissberg et al., 2015; Cristóvão et al., 2017; O’Conner et al., 2017). However, a multitude of frameworks and terminology related to SEL can be found in the literature, sometimes conflicting as different disciplines may use different terms to define SEL skills (Jones et al., 2019). This has raised several concerns, such as the risk of misinterpreting or over-generalizing outcomes (Jones et al., 2016). In response to this challenge, and with the goal of helping researchers, practitioners, and policymakers make sense of frameworks and related terminology to define and describe SEL skills, the Taxonomy Project developed an online resource (Explore SEL; http://exploresel.gse.harvard.edu), which provides a scientifically grounded system to explore, compare, and connect different SEL frameworks.

Since children spend a large amount of time in formal education settings, including preschools, this can be one of the pivotal settings for implementing SEL and supporting children's development of social and emotional skills (e.g., Jones and Bouffard, 2012; Domitrovich et al., 2017; Mahoney et al., 2020). In fact, previous research indicates that SEL can be incorporated into routine educational practices and do not require outside personnel for their effective delivery (Durlak et al., 2011; Taylor et al., 2017). Therefore, formal education settings and teachers are encouraged to integrate the teaching and reinforcement of SEL skills into their daily interactions and practices with children for creating safe and supportive learning environments and promoting social and emotional skills (e.g., Weissberg et al., 2015; Bierman et al., 2016).

Considering the goal of improving all children's health and development, and while SEL skills are not seen as a core part of the educational system's agenda, several authors have emphasized the benefits of universal SEL interventions (Greenberg et al., 2017; Mahoney et al., 2020). An exclusive focus on children with higher levels of needs could entail risks of fragmentation and marginalization of SEL interventions in educational settings (e.g., Jones and Bouffard, 2012; Domitrovich et al., 2017; Mahoney et al., 2020; Murano et al., 2020). Compelling empirical evidence from research systematic reviews (e.g., Catalano et al., 2004) and meta-analysis (e.g., Durlak et al., 2011; Taylor et al., 2017) has documented the efficacy of highquality, school-based, universal SEL programs, although most research has been conducted in the United States and with elementary and older grade students (Bierman and Motamedi, 2015; Gershon and Pellitteri, 2018).

In their landmark meta-analysis of quasi-experimental and experimental studies, Durlak and colleagues (2011) included 213 universal SEL programs implemented among kindergarten through high school students (27 outside the United States). They showed that SEL interventions seemed to have a significant positive impact on students' social and emotional skills and attitudes, as well as on behavior adjustment. Moreover, they found that students who participated in SEL programs improved their academic scores significantly, compared to control groups. More recently, Taylor and colleagues (2017), extended the findings of Durlak et al. (2011) by reviewing 82 universal school-based SEL programs (38 outside the United States), delivered within K-12 settings, with the main purpose of analyzing their long-term effects. Follow-up outcomes, collected up to 18 years after intervention, demonstrated significant enhancements in the participant groups on social-emotional skills, but also on academic performance, emotional distress and drug use, compared to controls. However, findings from school-based studies cannot be readily translated to ECEC settings given the key developmental tasks facing our youngest children (e.g., Bierman and Motamedi, 2015; Jones and Doolittle, 2017; Denham, 2018; Mahoney et al., 2020). As mentioned by 
Bierman and Motamedi (2015), SEL interventions in preschool settings need special consideration regarding contents, instructional approaches as well as opportunities to practice skills. Denham (2018) also underlines that SEL programs for these ages must involve more play and be less group-oriented than those for older children.

Previous research underlines the importance of early and preschool years for human development (Council for Early Child Development, 2010; Alfonso and DuPaul, 2020), the sensitive period and window of opportunities at this age (Zeanah et al., 2011; OECD, 2017), and the premise of returned investments made in early and preschool years (Naudeau et al., 2011; Shonkoff, 2017; Heckman, 2021a; Heckman, 2021b). Despite the large number of primary studies and several systematic reviews on the effects of SEL interventions on school aged children, there seems to be a lack of conclusive knowledge on the effects of SEL intervention on younger children (i.e., the pre-school years) and just a few have focused exclusively on universal evidence-based programs. Considering the accumulated evidence on the importance of ECEC to incorporate such programs in their curricula and daily practices, our study aims to contribute to the field by mapping what is known about the effects of universal SEL interventions for children under seven years of age in ECEC settings.

This overview aims to: 1 ) identify existing systematic reviews on universal, curriculum-based SEL interventions in preschool settings, assess their risk of bias, describe their characteristics and 2) synthesize the findings of the reviews with high methodological quality, and 3) identify knowledge gaps in practice relevant questions in the SEL domain.

\section{MATERIALS AND METHODS}

\section{Study Design}

This is a systematic review of systematic reviews based on quantitative studies published in peer-reviewed journals.

\section{Population, Interventions, Control, Outcomes}

The following PICO criteria were used in the literature search:

- Population: children aged 0-7 years.

- Interventions: universal programs with explicit intent to teach SEL skills, conducted in typical ECEC settings, i.e., not in high-risk or special education settings

- Control: experimental or quasi-experimental design

- Outcomes: efficacy and effectiveness of intervention as measured by child outcome data

\section{Search Strategy}

The original literature search was made on March 12, 2020, in seven different databases: CINAHL Complete, PsychINFO, PubMed, SocINDEX, ERIC, embase, and Scopus. The electronic search was supplemented using "Snowball methods", screening key references for additional literature. There were no language restrictions, but only English search terms were used. An example of the search strategy is provided in Supplementary Table 1 in the Supplement.

\section{Data Sources, Studies Sections, and Data Extraction}

Abstracts identified in the literature search according to the inclusion criteria were examined independently by two of the authors (DD and $\mathrm{BH}$ ). Articles were included if at least one author found the abstract potentially relevant, and the full text was studied. The full texts were divided into four equally large groups and examined in relation to the inclusion criteria by the whole author group independently in pairs. Disagreements between coders were resolved through mediation. Reviews which did not meet the inclusion criteria were excluded. Data extraction was performed independently by $\mathrm{DD}$ and $\mathrm{BH}$. Diverging results were discussed and resolved.

\section{Data Analysis}

The quality of the included reviews was assessed independently by two authors (DD and $\mathrm{BH}$ ) using the ROBIS tool (Whiting et al., 2016) as an assessment of risk of bias. ROBIS covers four domains to detect bias in systematic reviews relating to: study eligibility criteria; identification and selection of studies; data collection and study appraisal; synthesis and findings. The risk of bias of the systematic reviews was described according to the ROBIS assessment as "high," "low," or "unclear." Here too, disagreement was resolved through discussion between the two reviewers.

\section{RESULTS}

As summarized in the flowchart below (Figure 1), only two of the 105 publications reviewed in full text were deemed fit in accordance to previously stated inclusion criteria. Most publications had multiple reasons for exclusion (e.g., studies without a control group and interventions without explicit SEL interventions). A fair number of studies didn't meet the population-criteria as they either focused on school-aged children or included both school-aged and younger children. A complete list of the publications reviewed in full text along with their reasons for inclusion and exclusion is detailed in Supplementary Table 2 in the Supplement.

The two included reviews are presented in Table 1. Both reviews conducted a systematic review and meta-analysis regarding SELinterventions in early childhood care and education. Specifically, Blewitt et al. (2018) defined the target population as children aged 2-6 years in ECEC settings, while Luo et al. (2020) specified that the average child age had to be between 36-60 months at the start of the intervention conducted in a typical preschool setting.

\section{Search Strategy, Selection, Data Extraction and Analysis}

The included reviews were similar in objective, scope, procedure, and conclusion. A corrected covered area (CCA) of $25.53 \%$ was 


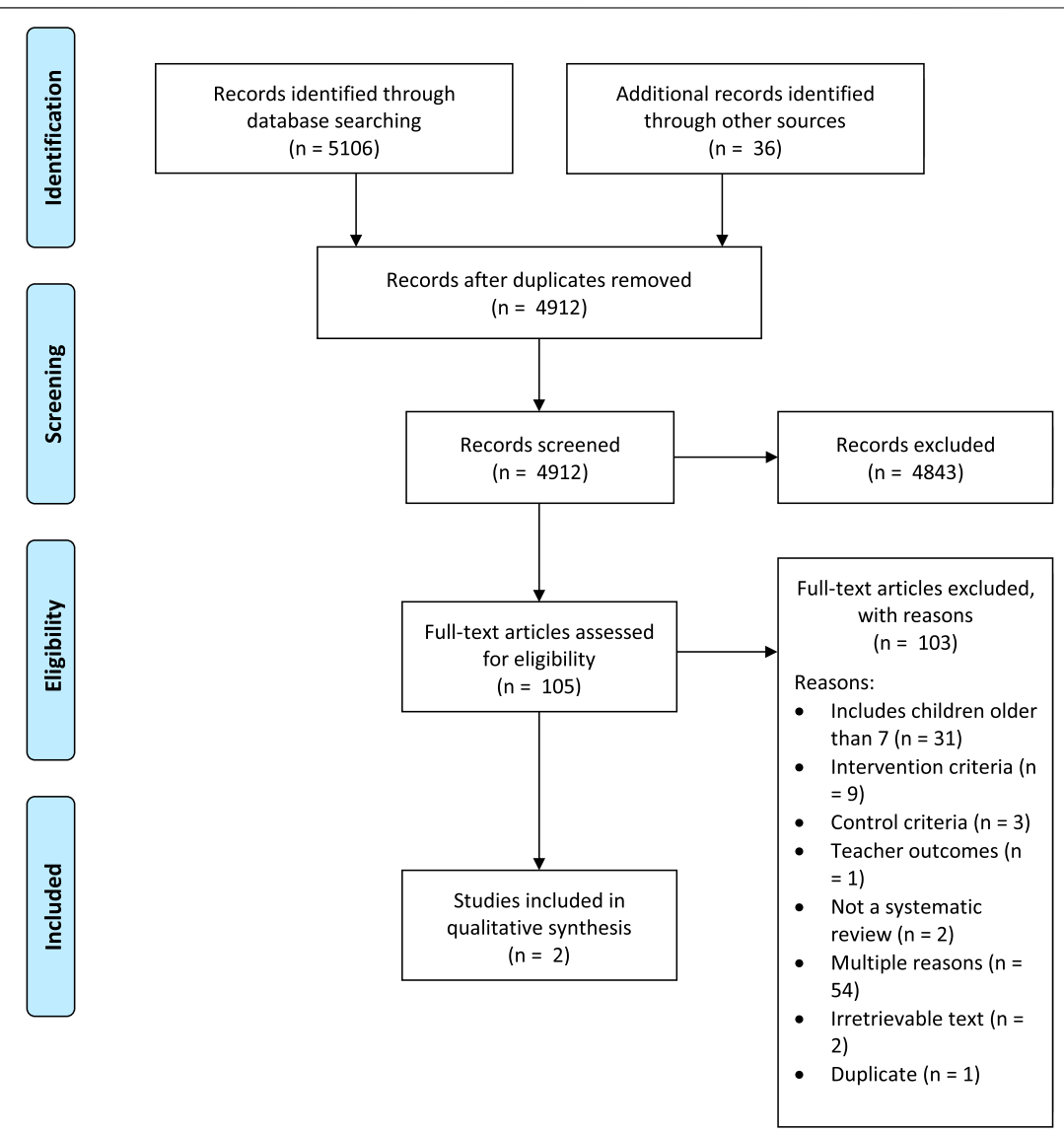

FIGURE 1 | PRISMA flow diagram.

found for the two reviews, which is considered very high $(>15)$ according to guidelines offered by Pieper et al. (2014). A detailed citation matrix is presented in Table 2.

However, some differences emerged. Luo et al. (2020) identified and screened more than twice the number of records $(n=30361)$ compared to Blewitt et al. (2018) $(n=$ 13 035). The number of full-text articles assessed for eligibility were similar (Blewitt et al., 2018, $n=362$ vs. Luo et al., 2020, $n=$ 379) but in the final stage, Blewitt et al. (2018) included approximately twice the number of studies $(n=79)$ compared to Luo et al. (2020) $(n=39)$. The differences could be attributed to diverging choices of databases where Blewitt et al. (2018) included a database covering biomedical and health research (MEDLINE Complete), while Luo et al. (2020) chose to search the multidisciplinary Academic Search premier, which does not have a medical focus, and the Education Full Text database, which covers education and related fields of research. In terms of PICO, Blewitt et al. (2018) specified a slightly broader age range for inclusion. Twenty-four of the non-overlapping studies included by Blewitt et al. (2018) included children outside of the age range specified by Luo et al. (2020), 21 of these studies included children above the age range of Luo and colleagues. In addition, Blewitt et al. (2018) included gray literature, which also accounts for some of the non-overlapping articles. The rest of the non-overlapping articles are likely missed or assessed differently by either review in relation to their inclusion criteria.

Both reviews reported interventions primarily from North America. Blewitt et al. (2018) reports approximately 65\%, while Luo et al. (2020) reports $72 \%$ of studies as being located in North America, respectively. Interventions were most often delivered by teachers, with $67 \%$ of studies reported by Blewitt et al. (2018) and $74 \%$ of studies reported by Luo et al. (2020), respectively, as containing teacher-directed interventions. Blewitt et al. (2018) reports that interventions typically occurred within the context of classroom activities using developmentally appropriate teaching methods. Luo et al. (2020) reports that $62 \%$ of primary studies did not specify the classroom activities in which the intervention was delivered. The rest were either delivered within whole group activities (26\%) or embedded within daily activities/routines (15\%). Reports regarding other intervention characteristics, such as core components, specific practices, and theoretical underpinnings, varied. Blewitt et al. (2018) summarizes a few common characteristics among included interventions, such as explicit and active instruction, modeling, opportunities for practice and reinforcement. The interventions vary in their application of these practices, underlying mechanisms of change and subsequent targeted skills. Luo et al. (2020) did not report a summary of corresponding intervention characteristics. 
TABLE 1 | Study characteristics of included reviews.

Blewitt et al. (2018)

(1) what social, emotional, behavioral, and early learning outcomes have been achieved by universal curriculum- based SEL interventions implemented in ECEC settings? (2) what program-level characteristics are associated with positive outcomes? (3) what are the methodologic limitations of research investigating the outcomes achieved by curriculumbased SEL interventions in ECEC settings?

Years 1995-2017, no language limits, peer-reviewed literature search conducted in ERIC, MEDLINE complete and PsycINFO. Gray literature searched via proquest dissertations and theses global database

79 (63 in meta-analysis)

18292 participants in 79 studies

Children aged 2-6 years/center-based ECEC setting

Universal curriculum-based SEL program (ie, included explicit teaching of SEL skills). The primary stated purpose of the SEL program was to increase children's social-emotional skill development

Experimental or quasi-experimental design (i.e., studies that did not or were not able to randomly allocate participants to intervention and control groups) with a control group

Each study was assessed against the effective public health practice project quality assessment tool for quantitative studies (Thomas et al., 2004) with respect to selection bias, study design, confounders, blinding, data collection methods, withdrawals, dropouts, intervention integrity, and analyses

Analysis of 1. the mean effect size across all studies and across each outcome category (cohen d). Heterogeneity of effect sizes was assessed using the intraclass correlation (ICC), Q-statistic and $l^{2}$ and $\tau 2$ tests. 2. a meta-regression was performed when ICC values were greater than 0.25 to examine the moderation effect of study-level characteristics

Overall $d=0.38(95 \% \mathrm{Cl}=0.24,0.51 ; p<0.001)$. Social competence $d=$ $0.30(95 \% \mathrm{Cl}=0.18,0.42 ; p<0.001)$. Emotional competence $\mathrm{d}=0.54$ $(95 \% \mathrm{Cl}=0.22,0.86 ; p<0.001)$. Behavioral and emotional difficulties $d=$ $0.19(95 \% \mathrm{Cl}=0.11,0.28 ; p<0.001)$. Self-regulation $d=0.28(95 \% \mathrm{Cl}=$ $0.11,0.46 ; p<0.001)$. Early learning outcomes $d=0.18(95 \% \mathrm{Cl}=0.02$, $0.33 ; p=0.03$ )

SEL programs administered at a relatively low intensity may be an effective way to increase social competence, emotional competence, behavioral self-regulation, and early learning outcomes and reduce behavioral and emotional difficulties in children aged 2-6 years. The SEL interventions appear to be particularly successful at increasing emotional knowledge, understanding, and regulation
Research question 1: What were the attributes of study participants and interventions involved in the review?

Research question 2: Did classroom-wide social-emotional interventions yield statistically significant and noteworthy mean effects for preschool children's social, emotional, and behavioral outcomes? Research question 3: Did select study or intervention characteristics moderate obtained intervention effects?

No filters/limits during electronic search conducted December 2015 (updated January 2018). Search conducted in academic search premier, ERIC, PsycINFO, academic full text

39 (33 in meta-analysis)

10646 participants in 39 studies

Children aged 3-5 years (36-60 months) on average at intervention-start, typical preschool setting

Classroom-wide social-emotional intervention defined as a curriculum, multicomponent intervention, hierarchical intervention, or intervention package/program intended for use with a whole class or groups of children in a class and designed to provide universal supports for improving the social, emotional, and behavioral outcomes of preschool children. No comprehensive/multiple domain curriculum and no secondary/tertiary interventions

Controlled group experimental design, specifically, a study design comparing the effects of the intervention between one group of participants who received the intervention to another group who did not experience the intervention, regardless of randomization

Cochrane's risk of bias tool (Higgins and Altman, 2008) with considerations for non-randomized studies (Reeves et al., 2013). Nine domains of risk of bias were examined: Sequence generation, allocation concealment, blinding of participants and personnel, blinding of outcome assessment, incomplete outcome data, selective outcome reporting, protection against contamination, baseline measurements, and procedural fidelity. Each domain was coded as low (low risk of bias), high (high risk of bias), or unclear (unclear risk of bias)

Analysis of 1. the mean effect sizes with a correction for small sample bias (Hedges'g). Heterogeneity was estimated using the Q-statistic and $l^{2}$. 2. Moderator analyses were conducted using a method analogous to the one-way analysis of variance for categorical variables and meta-regression for continuous variables. Examined the influence of one covariate at a time by conducting a univariate meta- regression analysis analogous to a simple regression for continuous variables

Social competence $\mathrm{g}=0.42(95 \% \mathrm{Cl}=0.28,0.56 ; p<0.001)$. Emotional competence $\mathrm{g}=0.33(95 \% \mathrm{Cl}=0.10,0.56 ; p=0.004)$. Challenging behavior $\mathrm{g}=-0.31(95 \% \mathrm{Cl}=-0.43,-0.19 ; p<0.001)$

Classroom-wide social-emotional interventions produce positive effects on the social, emotional, and behavioral outcomes of preschool children. Our findings suggest that these universal interventions are more efficacious when parents also are supported to implement universal strategies in the home settings
Objectives

Search information No. of studies included Population

Intervention

Control

Risk of bias tool used

Methods of analysis

Outcome

Conclusion
In Blewitt et al. (2018) only $16 \%$ of the primary studies were rated as high quality ( $44 \%$ moderate and $40 \%$ poor quality) and much of the downgrading was attributed to lack of blinding. Luo et al. (2020) did not report overall scores for quality assessments but detailed several areas where a substantial number of studies had a high or unclear risk of bias. They reported that no studies had managed to design a study were personnel and participants were blinded to the assigned condition. Most studies did not blind the outcome assessment either. They also assessed most studies as having an unclear risk of bias regarding sequence generation, i.e., the method by which participants were assigned. Furthermore, most primary studies did not report data on procedural fidelity. Additionally, they report that nearly half of the included primary studies were rated as unclear risk for incomplete outcome data (attrition bias) and protection against contamination (performance bias). 
TABLE 2 | Overlap of primary studies in included reviews.

\begin{tabular}{|c|c|c|}
\hline First author (year) & Blewitt (2018) & Luo (2020) \\
\hline Allen (2009) & $x$ & $x$ \\
\hline Amesty (2009) & $x$ & - \\
\hline Anliak (2010) & $\mathrm{x}$ & - \\
\hline Anticich (2013) & $x$ & - \\
\hline Aram (2008) & $\mathrm{x}$ & - \\
\hline Arda (2012) & $x$ & - \\
\hline Ashdown (2011) & $x$ & - \\
\hline Barnett (2008) & $x$ & - \\
\hline Bassett (2008) & $x$ & - \\
\hline Benitez (2011) & $x$ & $x$ \\
\hline Bierman (2008) & $x$ & - \\
\hline Boyle (2008) & $x$ & - \\
\hline Brigman (1999) & $x$ & - \\
\hline Brigman (2003) & $\mathrm{x}$ & - \\
\hline Carpenter (2002) & $\mathrm{x}$ & - \\
\hline Conner (2011) & $x$ & $x$ \\
\hline Deacon (2012) & $x$ & - \\
\hline Denham (1996) & $x$ & $x$ \\
\hline Dereli (2009) & $x$ & - \\
\hline Dereli-Iman (2014) & $x$ & - \\
\hline Dobrin (2013) & $x$ & - \\
\hline Domitrovich (2007) & $x$ & $x$ \\
\hline Dubas (1998) & $x$ & $x$ \\
\hline Fishbein (2016) & $x$ & - \\
\hline Flook (2015) & $x$ & $x$ \\
\hline Garrison (2017) & $x$ & - \\
\hline Gavazzi (2011) & $x$ & - \\
\hline Giménez-Dasí (2015) & $x$ & - \\
\hline Gunter (2012) & $x$ & $x$ \\
\hline Hall (2008) & $x$ & - \\
\hline Hamre (2012) & $x$ & $x$ \\
\hline Han (2005) & $x$ & $x$ \\
\hline Hughes (2015) & $x$ & $x$ \\
\hline Izard (2004) & $x$ & $x$ \\
\hline Izard (2008) & $x$ & $x$ \\
\hline Jack (2009) & $x$ & - \\
\hline Jakob (2005) & $x$ & - \\
\hline Justicia-Arráez (2015) & $x$ & - \\
\hline King (2001) & $x$ & - \\
\hline Koglin (2011) & $x$ & - \\
\hline Landry (2014) & $x$ & - \\
\hline Larmar (2006) & $x$ & - \\
\hline Lewis (2012) & $x$ & - \\
\hline Lonigan (2015) & $x$ & - \\
\hline Lösel (2006) & $x$ & - \\
\hline Lynch (2004) (Michigan study) & $x$ & $\mathrm{x}$ \\
\hline McKinney (1998) & $x$ & - \\
\hline Mishara (2006) & $x$ & - \\
\hline Moisan (2014) & $x$ & - \\
\hline Morris (2014) & $x$ & - \\
\hline O'Connor (2014) & $x$ & - \\
\hline Opre (2013) & $x$ & - \\
\hline Ornaghi (2017) & $x$ & - \\
\hline Ornaghi (2015) & $x$ & - \\
\hline Ostrov (2015) & $x$ & - \\
\hline Pahl (2010) & $x$ & $x$ \\
\hline Petermann (2008) & $x$ & - \\
\hline Pickens (2009) & $x$ & $x$ \\
\hline Poehlmann-Tynan (2016) & $x$ & - \\
\hline Randall (2011) & $x$ & - \\
\hline Reid (2007) & $x$ & - \\
\hline Rodker (2013) & $x$ & - \\
\hline Saltali (2010) & $x$ & - \\
\hline Sandy (2000) & $x$ & $x$ \\
\hline
\end{tabular}

TABLE 2 | (Continued) Overlap of primary studies in included reviews.

\begin{tabular}{|c|c|c|}
\hline First author (year) & Blewitt (2018) & Luo (2020) \\
\hline Schell (2015) & $x$ & - \\
\hline Schmitt (2014) & $x$ & - \\
\hline Schmitt (2017) & $x$ & - \\
\hline Serna (2000) & $x$ & $x$ \\
\hline Serna (2003) & $x$ & $x$ \\
\hline Seyhan (2017) & $x$ & $x$ \\
\hline Starnes (2017) & $x$ & - \\
\hline Stefan (2013) & $x$ & $x$ \\
\hline Stephenson (2009) & $x$ & - \\
\hline Tominey (2011) & $x$ & - \\
\hline Ulutaş (2007) & $x$ & - \\
\hline Upshur (2017) & $x$ & $x$ \\
\hline Upshur (2013) & $x$ & $x$ \\
\hline Vestal (2001/2004) & $x$ & $x$ \\
\hline Webster-Stratton (2008) & $x$ & - \\
\hline Webster-Stratton (2001) & - & $x$ \\
\hline Baker-Henningham (2009) & - & $x$ \\
\hline Feil (2009) & - & $x$ \\
\hline Feis (1985) & - & $x$ \\
\hline Finlon (2015) & - & $x$ \\
\hline Fossum (2017) & - & $x$ \\
\hline Giménez-Dasí (2017) & - & $x$ \\
\hline Hemmeter (2016) & - & $x$ \\
\hline Hutchings (2013) & - & $x$ \\
\hline Kayllı (2016) & - & $x$ \\
\hline Lynch (2004) (Virginia study) & - & $x$ \\
\hline Morris (2013) & - & $x$ \\
\hline Ostrov (2009) & - & $x$ \\
\hline Shure (1982) & - & $x$ \\
\hline Ştefan (2008) & - & x \\
\hline Corrected covered area (CCA) & $25,53 \%$ & Very high $(>15)$ \\
\hline
\end{tabular}

There were separate outcomes for social competence and emotional competence in both reviews. They both also included an overall outcome for externalizing and internalizing problem behavior called "behavioral and emotional difficulties" and "challenging behavior", respectively. Beyond this, Blewitt et al. (2018) calculates effect sizes for early learning outcomes and behavioral self-regulation, which Luo et al. (2020) does not. It is unclear to what degree the two reviews overlap in what measurements comprise the aggregated outcomes, since Blewitt et al. (2018) does not report what subscales are used (in applicable cases). Note that direct comparisons between the effect sizes should be avoided. While both reviews report aggregated standardized mean differences between posttest scores, there are differences in the meta-analytical procedures. Blewitt et al. (2018) calculated effect sizes using Cohen's $d$ while Luo et al. (2020) opted for Hedges' $g$. Blewitt et al. (2018) also reports a procedure for factoring in baseline differences. There is also a mention and citation in regard to taking nesting and nonindependence of multiple measures into account, though the exact procedure is not specified. Luo et al. (2020) reports a procedure in choosing one measurement when facing multiple choices in order to adhere to the assumption of statistical independence. Thus, there are differences, both accounted for and unaccounted, in the included primary studies (e.g., different interventions, population and outcomes) and meta-analytical procedures that make direct comparison of outcomes uncertain and likely inaccurate. 


\section{Overall Outcome of SEL Interventions}

Only one review (Blewitt et al., 2018) reported the overall outcome of program participation. The overall mean effect size for 391 included effects was Cohen $d=0.38$ (95\% CI, $0.24-0.51 ; p<0.001)$.

\section{Outcome in Social Competence}

From 34 effects, Luo and colleagues (2020) reported a small to medium mean effect size of the interventions, using Hedges'g, on the social competence of preschool children $[\mathrm{g}=0.42$ (CI, 0.28-0.56); $p<0.001]$. Blewitt and colleagues reported a similar mean (based on 115 effects) effect size in the social competence category [Cohen $\mathrm{d}=0.30$ (CI, 0.18-0.42); $p<0.001$ ].

\section{Outcome in Emotional Competence}

A medium to large mean effect size [Cohens $\mathrm{d}=0.54$ (CI, 0.22-0.86); $p<0.001$ ] was seen in Blewitt et al. (2018) on measures of emotional competence (based on 54 effects). Luo et al. (2020) found a somewhat lower mean effects size (14 comparisons) on emotional competence $[\mathrm{g}=0.33$ (CI, 0.10-0.56); $p=0.004$ ].

\section{Other Outcomes}

In the review by Luo and colleagues (2020) there was a significant reduction of what they named "challenging behavior" $[\mathrm{g}=-0.31$ (CI, $-0.43-0.19) ; p<0.001]$ based on 28 comparisons. Blewitt and colleagues (2018) reported small but significant effects in a domain (170 effects) called "behavioral and emotional difficulties" [ $\mathrm{d}=0.19$ (CI, 0.11-0.28); $p<0.001]$ and a similar mean effect size (based on 16 effects) in an area called "selfregulation" [ $\mathrm{d}=0.28$ (CI, 0.11-0.46); $p<0.001$ ]. Finally, Blewitt and colleagues (2018) also reported an outcome called "early learning outcome" where 36 comparisons had a mean effect size of 0.18 (CI, 0.02-0.33); $p=0.03$ ).

\section{Risk of Bias}

Using ROBIS, we found no systematic review with a low risk of bias. The two included systematic reviews were both judged to have high risk of bias. Both reviews used predefined protocols and we found few risks related to their specification of study eligibility criteria. The selection process and collection of data were less transparent and thorough in Blewitt and colleagues (2018) compared to Luo et al. (2020), which entails a risk of bias. Concerning the methods used to synthesize results, both reviews had a high risk of bias. Major risks were found in both reviews concerning the synthesizing of results. There is a lack of analysis and inference of how attrition might affect the results and despite considerable issues with high between-study variation (heterogeneity) in the primary studies and problems with robustness this is not included in the overall conclusion of the reviews. A more detailed outlining of the ROBIS-assessment is provided in Supplementary Table 3 in the Supplement.

\section{DISCUSSION}

The purpose of this mapping survey was to identify, assess and synthesize existing systematic reviews on universal pre-school interventions for increasing the social and emotional skills of children aged seven or younger. We also aimed to identify knowledge gaps in the SEL area relevant for ECEC practice. After screening nearly 5,000 records, only two reviews were found eligible for inclusion. Together, these reviews analyzed the findings from more than 90 primary studies, the vast majority studying at least one unique intervention.

Among the records initially screened for inclusion many were excluded due to the age criteria. SEL interventions in schools are well studied. However, generalizing effects from school to ECEC could be problematic due to developmental and organizational differences between the two contexts, which call for unique or adapted pre-school SEL-interventions. The relative scarcity of well-designed studies on SEL in preschool settings is somewhat surprising. Schools prioritize learning outcomes (e.g., literacy) and might face more difficulties in scheduling and implementing SEL-interventions within the academic curricula, compared to ECEC contexts where a balance between activities focusing on play, pre-academic skills and self-regulation could be appropriate (Slot et al., 2016). While curricula and organizational goals may vary among preschools (OECD, 2017), there are no obvious reasons why preschools should not be able to implement, and study, early and wide-reaching prevention-programming through high-quality SEL. Though it is beyond the scope of this review to analyze the reasons behind this lack of research, future studies should for example explore the importance of intervention design, meeting the needs of the broad range of developmental levels present in preschool children, in relation to other obstacles to implementation and growth of an evidencebased practice in this area. While some childhood interventions have demonstrated long-term effects, despite being provided in a limited timeframe (e.g., Bierman et al., 2020), the potential of a continuous prevention, throughout all stages of childhood, is depending on this challenge.

The two included systematic reviews were largely similar in their research questions and how they sought to answer them. There were, however, a few noteworthy differences. Most prominently, the number of included primary studies in each review differed considerably. Most of these non-overlapping studies could possibly be attributed to Blewitt et al. (2018) including a broader age range and gray literature as opposed to Luo et al. (2020). In addition, Blewitt et al. (2018) also searched a more medicine-oriented database which could potentially yield more relevant intervention studies. On the other hand, Luo et al. (2020) had a considerably greater initial yield following their search strategy. Considering the similarities in PICOs between the two reviews, the reasons for many non-overlapping studies remain unaccounted for, possibly attributable to differences on a more detailed level regarding search strategy, review process and inclusion criteria (Hennessy and Johnson, 2020). While the CCA is at a very high level, sharing more than a quarter of the primary studies, the reviews are similar enough and conducted in such close temporal proximity, that there is reason to wonder why the overlap wasn't on an even higher level.

This discrepancy illustrates a potentially larger issue, reflected in the risk of bias assessment. Both reviews were judged to have an overall high risk of bias. This is partly due to the broad and 
somewhat ill-defined nature of the field itself where there is a lack of agreement of what defines social and emotional learning, i.e., what interventions and outcomes comprise the construct of SEL. This is likely to impact any attempt to identify and synthesize primary studies regarding SEL, e.g., the formulation of search criteria, the assessment of eligibility and the categorization of outcomes. Though both reviews reported which measurement scales were included in which aggregated outcome, the outcomes themselves lacked pre-defined specificity. For example, Blewitt et al. (2018) used an outcome called "Behavioral and emotional difficulties", and Luo et al. (2020) constructed an outcome called "Challenging behavior". Both these constructs combined various scales measuring both internalizing and externalizing problem behavior. Incompatibilities such as these induces additional risks of bias. The choice of appropriate and meaningful outcomes when studying SEL-interventions is another related issue which also remains subject to interpretation. For example, only Blewitt et al. (2018) included behavioral self-regulation as an aggregated outcome. While there could be several reasons behind this disparity, conceptual clarity could further any prospective attempts to synthesize SEL-interventions and their effects. This echoes previous calls for increased precision in defining SELconstructs in research (e.g., Jones et al., 2016).

The included reviews also carried some individual strengths and weaknesses regarding risks of bias. While Luo et al. (2020) utilized multiple independent assessors for assessing eligibility, conducting data extraction, and assessing risk of bias, Blewitt et al. (2018) did not utilize this strategy consistently. While not always a notable risk, human error and different interpretations are still possible and remain important in as broad a field as SEL. Multiple independent assessors with methods for resolving discrepancies is one way of potentially reducing the inherent risks, though it carries the drawback of increased costs. Additionally, there were some differences in how the reviews detailed the inclusion criteria. Luo et al. (2020) reported examples and non-examples of interventions in relation to universal supports and at-risk children, in addition to specifying and constraining the setting to typical preschools. Blewitt et al. (2018) did not provide a similarly detailed description of their inclusion criteria. This is important since the level of needs of the participants and the type of educational setting where the intervention is delivered, have a potentially large influence on the effect sizes.

In addition to the considerable heterogeneity regarding interventions and outcomes, the risk of bias in primary studies is also an outstanding issue when synthesizing studies about SEL. While Luo et al. (2020) assessed the risk of bias in several domains for the included studies, these results were not utilized further in the synthesis. This could induce risk in several ways. An expanded analysis (e.g., sensitivity analysis or moderator analysis) and discussion regarding risk of bias in primary studies could potentially lead to different conclusions. For instance, Luo et al. (2020) rated the majority of primary studies as unclear risk regarding baseline measurements in the allocation process (i.e., unclear whether the groups are comparable or not), meaning that it is unclear to what degree intervention effects could be attributable to inherent group differences. Blewitt et al. (2018) on the other hand, utilized their risk of bias assessment as a moderator. While this analysis wasn't statistically significant in the final model, the low proportion of high-quality studies still needs to be discussed. Otherwise, there is a risk of overemphasizing non-significance when there is a chance that different ratings, models of metaregression or sensitivity analyses could show different results. Overall, the issue could stand to be discussed further, seeing as it could affect several aspects of the synthesis beyond the final aggregated outcomes. Another issue in the synthesis was taking differences in baseline measurements into account. Blewitt et al. (2018) reports taking baseline differences into account, but Luo et al. (2020) only reports calculating effect sizes using the standardized mean difference between posttests. Significant baseline differences between the groups that are not considered could potentially affect the final calculations of effect sizes. In sum, considerable heterogeneity in study design, interventions, outcomes, risk of bias, effect sizes are reasons to look at alternative methods of quantitative synthesis, or even refraining from it altogether (Achana et al., 2014).

Looking at the included reviews, some additional common themes emerged. Most of the primary studies and subsequent interventions were located in North America, primarily the US. This raises questions concerning the generalizability of effects of SEL-programs when transferred to other geographical and cultural contexts. Future research should investigate, more thoroughly, how translation and adaptation of programs effects the outcomes. Both reviews also emphasized that in most included primary studies, the teacher was the designated intervention agent. This supports previous statements (Durlak et al., 2011; Taylor et al., 2017) that it is possible to integrate SELprogramming into educational curricula, with teachers playing a key role in its delivery. Regarding the interventions themselves, neither of the reviews presented clear descriptions of the contents of the activities, their theoretical underpinnings and which components of the intervention were responsible for the effect. If future primary studies used standardized protocols to support these analyses, it would be of major help to the scientific field of SEL.

A final remark is that Blewitt et al. (2018) and Luo et al. (2020) both reported positive results for all measured outcomes in favor of universal SEL-interventions in preschool. However, the high risks of bias found in both reviews would call upon a much more tentative interpretation of the effects of universal SELinterventions in preschool.

\section{Summary of Main Findings}

The main finding of this study is that very few systematic reviews has been published with a focus on the effects of universal SEL interventions on the social and emotional competence of young children in ECEC settings. The two identified reviews and their primary studies suffer from a number of scientific weaknesses and risks of bias leading us to the conclusion that the area must be considered a knowledge gap.

\section{Key Topics}

- The two included reviews had similar objectives and PICOs, with their searches conducted in close temporal proximity. 
This a likely explanation for the very high overlap in primary studies, still the reasons for a notable amount of the nonoverlapping primary studies remain unaccounted for.

- Most of the primary studies and interventions were conducted in North America. Teachers were most often the intervention agent. No clear themes emerged regarding what activities the interventions consisted of and what theoretical underpinnings they were based on.

- Both reviews were assessed to carry a high risk of bias. Most prominently due to their synthesis of heterogenous primary studies, with a considerable variation in areas such as: interventions, outcomes, study design, risk of bias, and effect sizes. In addition, synthesis is likely also made difficult due to the broad and abstract terms used to describe and operationalize SEL.

- Future research in the field of SEL may opt for greater precision and clarity in choosing and operationalizing constructs. Systematic reviews need to consider various forms of heterogeneity in synthesizing primary studies. There is a need for more high-quality primary studies.

\section{Limitations}

While measures were taken to formulate a PICO that was specific, but still congruent with the broad nature of the field, problems were still evident in assessing the eligibility of studies. In large, the issues mentioned previously regarding specificity and precision in SEL-related constructs apply here as well. We used generous search terms to minimize the risk of excluding important studies but there is still a risk that potentially interesting reviews have not been identified.

A potential limitation is the use of ROBIS as a tool for assessing the risk of bias in included systematic reviews. ROBIS offers a comprehensive, thorough, and structured way of assessing risk of bias, but while its application has been studied in the field of public health and biomedicine (e.g., Gates et al., 2018), studies regarding its applicability in social sciences remains limited.

\section{Conclusion and Future Directions}

We have described a knowledge gap in the area of universal SELinterventions in preschool settings. Researchers conducting primary studies and systematic reviews in this area are advised to increase precision in constructs and reduce the risk of bias to facilitate reliable conclusions. There is still a lack of well-designed, high-quality primary studies evaluating SEL-interventions for our youngest children. Future studies looking to aggregate outcomes through meta-analytic procedures could look at different options of reducing heterogeneity. One way is to streamline the PICO to look at more precise and possibly compartmentalized aspects of

\section{REFERENCES}

Achana, F., Hubbard, S., Sutton, A., Kendrick, D., and Cooper, N. (2014). An Exploration of Synthesis Methods in Public Health Evaluations of Interventions Concludes that the Use of Modern Statistical Methods Would Be Beneficial. J. Clin. Epidemiol. 67, 376-390. doi:10.1016/j.jclinepi.2013.09.018
SEL, both in terms of interventions and outcomes. Continued research in SEL would be much facilitated by more precise constructs. The Taxonomy project (Explore SEL; http:// exploresel.gse.harvard.edu), mentioned previously, is an example of a promising attempt to promote precision in SEL.

\section{DATA AVAILABILITY STATEMENT}

The original contributions presented in the study are included in the article/supplementary material, further inquiries can be directed to the corresponding author.

\section{AUTHOR CONTRIBUTIONS}

$\mathrm{DD}, \mathrm{NK}, \mathrm{AD}, \mathrm{AM}, \mathrm{CP}, \mathrm{VC}, \mathrm{MA}, \mathrm{EK}, \mathrm{FM}$, and $\mathrm{BH}$ assessed the relevance of full text articles, analyzed and interpreted the results, and participated in writing the manuscript. $\mathrm{DD}$ and $\mathrm{BH}$ designed the PICO and research questions, assessed the relevance of abstracts, conducted quality assessments with ROBIS and extracted data.

\section{FUNDING}

This paper is financed by the EU Erasmus + Program via the project "Social and Emotional Skills Development in Early Childhood Education and Care in Europe" (EU-SELF), 20191-BG01-KA201-062593, KA2 - Cooperation for innovation and the exchange of good practices, KA201 - Strategic Partnerships for school education.

\section{ACKNOWLEDGMENTS}

We are grateful for valuable help with the literature search from Maria Björklund and Krister Aronsson at the Faculty of Medicine, Lund University for their help with the literature search. We would also like to express our gratitude to Alexandra Snellman at CAMTÖ Center for Assessment of Medical Technology in Örebro for valuable advice and input regarding ROBIS assessments.

\section{SUPPLEMENTARY MATERIAL}

The Supplementary Material for this article can be found online at: https:/www.frontiersin.org/articles/10.3389/feduc.2021.691670/ full\#supplementary-material

Alfonso, V. C., and DuPaul, G. J. (2020). "Introduction: The Importance of Early Childhood Development, Education, and Intervention," in Healthy Development in Young Children: Evidence-Based Interventions for Early Education. Editors V. C. Alfonso and G. J. DuPaul (Washington, DC: American Psychological Association), 3-10. doi:10.1037/0000197-001

Berg, J., Nolan, E., Yoder, N., Osher, D., and Mart, A. (2019). Social-emotional Competencies in Context: Using Social-Emotional Learning Frameworks to 
Build Educators' Understanding. Available at: https://measuringsel.casel.org/ wp-content/uploads/2019/02/Frameworks-C.2-pdf [Accessed March 11, 2021].

Bierman, K. L., Greenberg, M. T., and Abenavoli, R. (2016). Promoting Social and Emotional Learning in Preschool: Programs and Practices that Work. Edna Bennet Pierce Prevention Research Center, Pennsylvania State University.

Bierman, K. L., Heinrichs, B. S., Welsh, J. A., and Nix, R. L. (2021). Reducing Adolescent Psychopathology in Socioeconomically Disadvantaged Children with a Preschool Intervention: A Randomized Controlled Trial. Am. J. Psychiatry 178, 305-312. doi:10.1176/appi.ajp.2020.20030343

Bierman, K. L., and Motamedi, M. (2015). "SEL Programs for Preschool Children," in Handbook on Social and Emotional Learning: Research and Practice. Editors J. A. Durlak, C. E. Domitrovich, R. P. Weissberg, and T. P. Gullotta (New York: Guilford), 135-150.

Bierman, K. L., Torres, M. M., Domitrovich, C. E., Welsh, J. A., and Gest, S. D. (2009). Behavioral and Cognitive Readiness for School: Cross-Domain Associations for Children Attending Head Start. Soc. Dev. 18, 305-323. doi:10.1111/j.1467-9507.2008.00490.x

Blewitt, C., Fuller-Tyszkiewicz, M., Nolan, A., Bergmeier, H., Vicary, D., Huang, T., et al. (2018). Social and Emotional Learning Associated with Universal Curriculum-Based Interventions in Early Childhood Education and Care Centers. JAMA Netw. Open 1, e185727. doi:10.1001/ jamanetworkopen.2018.5727

Catalano, R. F., Berglund, M. L., Ryan, J. A. M., Lonczak, H. S., and Hawkins, J. D. (2004). Positive Youth Development in the United States: Research Findings on Evaluations of Positive Youth Development Programs. ANNALS Am. Acad. Polit. Soc. Sci. 591, 98-124. doi:10.1177/0002716203260102

Council for Early Child Development. (2010). The Science of Early Child Development, Available at: http://mccahouse.org/wp-content/uploads/2014/ 12/Brochure_Science_of_ECD_June2010.pdf [Accessed March 10, 2021].

Cristóvão, A. M., Candeias, A. A., and Verdasca, J. (2017). Social and Emotional Learning and Academic Achievement in Portuguese Schools: A Bibliometric Study. Front. Psychol. 8, 1913. doi:10.3389/fpsyg.2017.01913

Denham, S. A., Bassett, H. H., Thayer, S. K., Mincic, M. S., Sirotkin, Y. S., and Zinsser, K. (2012). Observing Preschoolers' Social-Emotional Behavior: Structure, Foundations, and Prediction of Early School Success. J. Genet. Psychol. 173 (3), 246-278. doi:10.1080/00221325.2011.597457

Denham, S. A. (2018). Keeping SEL Developmental: The Importance of a Developmental Lens for Fostering and Assessing SEL Competencies. Measuring SEL: Using Data to Inspire Practice. Available at: https:// measuringsel.casel.org/wp-content/uploads/2018/11/Frameworks- DevSEL. pdf [Accessed March 13, 2021].

Domitrovich, C. E., Durlak, J. A., Staley, K. C., and Weissberg, R. P. (2017). Socialemotional Competence: An Essential Factor for Promoting Positive Adjustment and Reducing Risk in School Children. Child. Dev. 88, 408-416. doi:10.1111/cdev.12739

Durlak, J. A., Weissberg, R. P., Dymnicki, A. B., Taylor, R. D., and Schellinger, K. B. (2011). The Impact of Enhancing Students' Social and Emotional Learning: A Meta-Analysis of School-Based Universal Interventions. Child. Dev. 82, 405-432. doi:10.1111/j.1467-8624.2010.01564.x

Eklund, K., Kilpatrick, K. D., Kilgus, S. P., and Haider, A. (2018). A Systematic Review of State-Level Social-Emotional Learning Standards: Implications for Practice and Research. Sch. Psychol. Rev. 47, 316-326. doi:10.17105/SPR-2017.0116.V47-3

Gates, A., Gates, M., Duarte, G., Cary, M., Becker, M., Prediger, B., et al. (2018). Evaluation of the Reliability, Usability, and Applicability of AMSTAR, AMSTAR 2, and ROBIS: Protocol for a Descriptive Analytic Study. Syst. Rev. 7 (1), 1-7. doi:10.1186/s13643-018-0746-1

Gershon, P., and Pellitteri, J. (2018). Promoting Emotional Intelligence in Preschool Education: A Review of Programs. Int. J. Emot. Educ. 10 (2), 26-41. https://www.um.edu.mt/library/oar/handle/123456789/36464

Goldberg, J. M., Sklad, M., Elfrink, T. R., Schreurs, K. M. G., Bohlmeijer, E. T., and Clarke, A. M. (2019). Effectiveness of Interventions Adopting a Whole School Approach to Enhancing Social and Emotional Development: a Meta-Analysis. Eur. J. Psychol. Educ. 34, 755-782. doi:10.1007/s10212-018-0406-9

Greenberg, M. T., Domitrovich, C. E., Weissberg, R. P., and Durlak, J. A. (2017). Social and Emotional Learning as a Public Health Approach to Education. Future Child. 27 (1), 13-32. Available at: http://www.jstor.org/stable/44219019. doi:10.1353/foc.2017.0001
Heckman, J. (2021b). Invest in Early Childhood Development: Reduce Deficits, Strengthen the Economy. The Heckman Equation, Available at: https:// heckmanequation.org/resource/invest-in-early-childhood-development-reducedeficits-strengthen-the-economy/ [Accessed March 2, 2021].

Heckman, J., (2021a). There's More to Gain by Taking a Comprehensive Approach to Early Childhood Development. The Heckman Equation, Available at: https:// heckmanequation.org/resource/research-summary-lifecycle-benefits-influentialearly-childhood-program/ [Accessed Febr 9, 2021].

Hennessy, E. A., and Johnson, B. T. (2020). Examining Overlap of Included Studies in Meta-reviews: Guidance for Using the Corrected Covered Area index. Res. Syn Meth 11 (1), 134-145. doi:10.1002/jrsm.1390

Higgins, J. P. T., and Altman, D. G. (2008). "Assessing Risk of Bias in Included Studies," in Cochrane Handbook for Systematic Reviews of Interventions. Editors J. P. T. Higgins and S. Green (Chichester, West Sussex: Wiley), 187-242.

Jones, D. E., Greenberg, M., and Crowley, M. (2015). Early Social-Emotional Functioning and Public Health: The Relationship between Kindergarten Social Competence and Future Wellness. Am. J. Public Health 105, 112283-112290. doi:10.2105/AJPH.2015.302630

Jones, S. M., Bailey, R., Brush, K., Nelson, B., and Barnes, S. (2016). What Is the Same and what Is Different? Making Sense of the "Non-cognitive" Domain. Helping Educators Translate Research into Practice. Easel Lab, Harvard Graduate School of Education. . Available at: https://easel.gse.harvard.edu/files/gse-easel-lab/files/ words_matter_paper.pdf. doi:10.5040/9781474282932.0014 (Accessed March $8,2021)$.

Jones, S. M., Bailey, R., Brush, K., and Nelson, B. (2019). Introduction to the Taxonomy Project: Tools for Selecting and Aligning SEL Frameworks. Establishing Practical Social-Emotional Competence Assessments Work Group: Collaborative for Academic, Social, and Emotional Learning. Available at: https://measuringsel.casel.org/wp-content/uploads/2019/02/ Frameworks-C.1.pdf [Accessed March 11, 2021].

Jones, S. M., and Bouffard, S. M. (2012). Social and Emotional Learning in Schools: From Programs to Strategies and Commentaries. Soc. Pol. Rep. 26 (4), 1-33. doi:10.1002/j.2379-3988.2012.tb00073.x

Jones, S. M., and Doolittle, E. J. (2017). Social and Emotional Learning: Introducing the Issue. Future Child. 27 (1), 3-11. doi:10.1353/foc.2017.0000

Luo, L., Reichow, B., Snyder, P., Harrington, J., and Polignano, J. (2020). Systematic Review and Meta-Analysis of Classroom-wide Social-Emotional Interventions for Preschool Children. Top. Early Child. Spec. Educ., 027112142093557. doi:10.1177/0271121420935579

Mahoney, J. L., Weissberg, R. P., Greenberg, M. T., Dusenbury, L., Jagers, R. J., Niemi, K., et al. (2020). Systemic Social and Emotional Learning: Promoting Educational success for All Preschool to High School Students. Am. Psychol. doi:10.1037/amp0000701

McClelland, M. M., Tominey, S. L., Schmitt, S. A., and Duncan, R. (2017). SEL Interventions in Early Childhood. Future Child. 27 (1), 33-47. Available at: http://www.jstor.org/stable/44219020 (Accessed March 11, 2021). doi:10.1353/ foc.2017.0002

Moffitt, T. E., Arseneault, L., Belsky, D., Dickson, N., Hancox, R. J., Harrington, H., et al. (2011). A Gradient of Childhood Self-Control Predicts Health, Wealth, and Public Safety. Proc. Natl. Acad. Sci. U. S. A. 108 (7), 2693-2698. doi:10.1073/pnas.1010076108

Murano, D., Sawyer, J. E., and Lipnevich, A. A. (2020). A Meta-Analytic Review of Preschool Social and Emotional Learning Interventions. Rev. Educ. Res. 90 (2), 227-263. doi:10.3102/0034654320914743

Nakamichi, K., Nakamichi, N., and Nakazawa, J. (2021). Preschool Social-Emotional Competencies Predict School Adjustment in Grade 1. Early Child. Develop. Care 191 (2), 159-172. doi:10.1080/ 03004430.2019 .1608978

Naudeau, S., Kataoka, N., Valerio, A., Neuman, M. J., and Elder, L. K. (2011). Investing in Young Children: An Early Childhood Development Guide for Policy Dialogue and Project Preparation. World Bank. Available at: https:// openknowledge.worldbank.org/handle/10986/2525 [Accessed March 1, 2021]. doi:10.1037/e596922012-001

O'Conner, R., Feyter, J., Carr, A., Luo, J. L., and Romm, H. (2017). A Review of the Literature on Social and Emotional Learning for Students Ages 3-8: Characteristics of Successful Social and Emotional Learning Programs. Available at: https://files.eric.ed.gov/fulltext/ED572721.pdf [Accessed March 8, 2021]. 
OECD (2017). Starting Strong 2017: Key OECD Indicators on Early Childhood Education and Care. Paris: Starting Strong: OECD Publishing. doi:10.1787/ 9789264276116-en

Pieper, D., Antoine, S.-L., Mathes, T., Neugebauer, E. A. M., and Eikermann, M. (2014). Systematic Review Finds Overlapping Reviews Were Not Mentioned in Every Other Overview. J. Clin. Epidemiol. 67 (4), 368-375. doi:10.1016/j.jclinepi.2013.11.007

Prado, E. L., and Dewey, K. G. (2014). Nutrition and Brain Development in Early Life. Nutr. Rev. 72 (4), 267-284. doi:10.1111/nure.12102

Reeves, B. C., Higgins, J. P. T., Ramsay, C., Shea, B., Tugwell, P., and Wells, G. A. (2013). An Introduction to Methodological Issues when Including Nonrandomised Studies in Systematic Reviews on the Effects of Interventions. Res. Syn. Meth. 4 (1), 1-11. doi:10.1002/jrsm.1068

Shonkoff, J. P. (2017). Rethinking the Definition of Evidence-Based Interventions to Promote Early Childhood Development. Pediatrics 140 (6), e20173136. doi:10.1542/peds.2017-3136

Slot, P. L., Cadima, J., Salminen, J., Pastori, G., and Lerkkanen, M. K. (2016). Multiple case study in seven European countries regarding culture-sensitive classroom quality assessment. Available at: https://ecec-care.org/fileadmin/ careproject/Publications/reports/CARE_WP2_D2_3_Multiple_Case_study_ FINAL_REPORT.pdf [Accessed March 4, 2021].

Spencer, S. J., Korosi, A., Layé, S., Shukitt-Hale, B., and Barrientos, R. M. (2017). Food for Thought: How Nutrition Impacts Cognition and Emotion. NPJ Sci. Food 1, 1. doi:10.1038/s41538-017-0008-y

Taylor, R. D., Oberle, E., Durlak, J. A., and Weissberg, R. P. (2017). Promoting Positive Youth Development through School-Based Social and Emotional Learning Interventions: A Meta-Analysis of Follow-Up Effects. Child. Dev. 88, 1156-1171. doi:10.1111/cdev.12864

Thomas, B. H., Ciliska, D., Dobbins, M., and Micucci, S. (2004). A Process for Systematically Reviewing the Literature: Providing the Research Evidence for Public Health Nursing Interventions. Worldviews Evidence-Based Nurs. 1 (3), 176-184. doi:10.1111/j.1524-475X.2004.04006.x

Weissberg, R. P., Durlak, J. A., Domitrovich, C. E., and Gullotta, T. P. (2015). "Social and Emotional Learning: Past, Present, and Future," in Handbook on
Social and Emotional Learning: Research and Practice. Editors J. A. Durlak, C. E. Domitrovich, R. P. Weissberg, and T. P. Gullotta (New York: Guilford), 3-19.

Whiting, P., Savović, J., Higgins, J. P. T., Caldwell, D. M., Reeves, B. C., Shea, B., et al. (2016). ROBIS: A New Tool to Assess Risk of Bias in Systematic Reviews Was Developed. J. Clin. Epidemiol. 69, 225-234. doi:10.1016/ j.jclinepi.2015.06.005

Yates, T., Ostrosky, M. M., Cheatham, C. A., Fettig, A., Shaffer, L., and Santos, R. M. (2008). Research Synthesis on Screening and Assessing Social-Emotional Competence. Urbana, AZ: University of Illinois, Center of the Social Emotional Foundations for Early Learning.

Zeanah, C. H., Gunnar, M. R., McCall, R. B., Kreppner, J. M., and Fox, N. A. (2011). VI. Sensitive Periods. Monogr. Soc. Res. Child Develop. 76 (4), 147-162. doi:10.1111/j.1540-5834.2011.00631.x

Conflict of Interest: The authors declare that the research was conducted in the absence of any commercial or financial relationships that could be construed as a potential conflict of interest.

Publisher's Note: All claims expressed in this article are solely those of the authors and do not necessarily represent those of their affiliated organizations, or those of the publisher, the editors and the reviewers. Any product that may be evaluated in this article, or claim that may be made by its manufacturer, is not guaranteed or endorsed by the publisher.

Copyright (C) 2021 Djamnezhad, Koltcheva, Dizdarevic, Mujezinovic, Peixoto, Coelho, Achten, Kolumbán, Machado and Hofvander. This is an open-access article distributed under the terms of the Creative Commons Attribution License (CC BY). The use, distribution or reproduction in other forums is permitted, provided the original author(s) and the copyright owner(s) are credited and that the original publication in this journal is cited, in accordance with accepted academic practice. No use, distribution or reproduction is permitted which does not comply with these terms. 